\title{
Adaptive Noise Filtering for Accurate and Precise Diffusion Estimation in Fiber Crossings
}

\author{
Matthan W.A. Caan ${ }^{1}$, Ganesh Khedoe ${ }^{2}$, Dirk Poot ${ }^{2}$, Arjan den Dekker ${ }^{2}$, \\ Silvia Olabarriaga ${ }^{1}$, Kees Grimbergen ${ }^{1}$, Lucas van Vliet $^{2}$, and Frans Vos $^{1,2}$ \\ 1 Academic Medical Center, University of Amsterdam, NL \\ 2 Delft University of Technology, NL \\ m.w.a.caan@amc. uva.nl
}

\begin{abstract}
Measuring the diffusion properties of crossing fibers is very challenging due to the high number of model parameters involved and the intrinsically low SNR of Diffusion Weighted MR Images. Noise filtering aims at suppressing the noise while pertaining the data distribution. We propose an adaptive version of the Linear Minimum Mean Square Error (LMMSE) estimator to achieve this. Our filter applies an adaptive filtering kernel that is based on a space-variant estimate of the noise level and a weight consisting of the product of a Gaussian kernel and the diffusion similarity with respect to the central voxel. The experiments show that the data distribution after filtering is still Rician and that the diffusivity values are estimated with a higher precision while pertaining an equal accuracy. We demonstrate on brain data that our adaptive approach performs better than the initial LMMSE estimator.
\end{abstract}

\section{Introduction}

Diffusion Weighted Magnetic Resonance Imaging (DW-MRI) is hampered by a low signal to noise ratio (SNR) for high values for the diffusion weighting parameter $b$. As a consequence, diffusion model parameters may be estimated with low precision. An incorrect representation of the noise properties, particularly assuming a Gaussian instead of a Rician noise distribution in the DWIs, may render a biased signal model [1]. Noise filtering aims to increase the accuracy and precision of the estimated diffusivity. A sufficiently high spatial resolution permits the identification of locally homogeneous tissue regions in which averaging reduces the noise, but pertains the signal.

In previous work, the Linear Minimum Mean Square Error (LMMSE) estimator [2] was proposed to reduce Rician distorted MR data, including DW-MRI. In DW-MRI data, anisotropic smoothing was applied to reduce noise [3. An estimate of the noise level was obtained by extending the single tensor description with a Rician noise distribution and estimating the noise level by a Maximum Likelihood (ML) framework 4/5]. Rician noise reduction by spatial regularization [6]7. was used to limit the bias in estimates of a single tensor model. Alternatively, sequential anisotropic multichannel Wiener filtering allows the correction of the bias in the diffusivity estimates due to the Rician distribution of Diffusion

T. Jiang et al. (Eds.): MICCAI 2010, Part I, LNCS 6361, pp. 167 174, 2010

(C) Springer-Verlag Berlin Heidelberg 2010 
Weighted Images (DWIs) [8]. By modeling a Markov Random tensor Field, a joint optimization of the likelihood and a prior could be performed [9]. These methods have in common that they are applied to a single tensor model.

To date, relatively little attention is paid to filtering noise in fiber crossings. Crossing fiber orientations were more precisely reconstructed after filtering [8]. Estimating diffusion properties independently per crossing fiber bundle involves more model parameters and hence is inherently more susceptible to noise 10.

We propose a method for noise suppression in fiber crossings, which is also valid in voxels with a single fiber configuration. The technique is inspired by the LMMSE estimator introduced by Aja-Fernandez [2, but it contains two important improvements. First, the original Aja-Fernandez approach used an isotropic, uniform kernel, which mixes neighbouring tissues around transitions. We use an adaptive Gaussian kernel by only including voxels whose single tensors are similarly shaped. Consequently, the bias induced by the mixing is reduced. Second, the Aja-Fernandez filtering approach uses a global estimate of the noise level $\sigma$, while the noise level is known to be smoothly varying due to parallel imaging [5]. We perform an ML estimation of $\sigma$ per voxel by fitting a constrained dual tensor model.

The distribution of the data after LMMSE-filtering should be known in order to allow accurate parameter estimation. In our experiments we will emperically show that our model, assuming a Rician distribution, still adequately describes the data. It is then demonstrated that the variance in the estimated diffusion parameters is decreased while the bias remains low. Finally, we will compare our adaptive noise suppression scheme with the original Aja-Fernandez approach on brain data.

\section{Method}

Linear Minimum Mean Square Estimation (LMMSE)-filtering [2] has been proposed to reduce noise in Rician distributed MRI data. This closed-form solution considers the DWIs as realizations of stochastic processes. The local signal variance is reduced, steered by the estimated noise level. The estimate of the squared signal $\hat{A}^{2}$ equals

$$
\hat{A^{2}}=\left\langle M^{2}\right\rangle-2 \sigma^{2}+\left(1-\frac{4 \sigma^{2}\left(\left\langle M^{2}\right\rangle-\sigma^{2}\right)}{\left\langle M^{4}\right\rangle-\left\langle M^{2}\right\rangle^{2}}\right)\left(M^{2}-\left\langle M^{2}\right\rangle\right),
$$

in which $M$ represents the measured signal and $\langle\cdot\rangle$ the estimator of a sample's expectation value.The LMMSE-filter is applied in 3-D to each DWI independently. The variance of the noise, $\sigma^{2}$, was originally assumed constant in the entire field of view and estimated as the mode of the locally estimated signal variances. The expectation value of the sample is approximated by a weighted sum in a local neighbourhood $\eta,\langle I\rangle=\frac{1}{\sum d_{p}} \sum_{p \epsilon \eta} d_{p} I_{p}$. Initially, uniform weighting, i.e. $d_{p}=1 \forall p$, was proposed. 
In the following Section 2.1 we will introduce an ML approach involving a dual tensor model for improved estimation of the noise level $\sigma$. Section 2.2 will discuss an adaptive filtering kernel which is valid at fiber crossings.

\subsection{Maximum Likelihood Noise Level Estimation}

DW-MRI records the magnitude of the complex MR signal. The real and imaginary channels of the complex MR image are assumed to be independently affected by Gaussian noise, such that the DWIs are Rician distributed. Hence, the probability density function of a measured signal $S_{m, j} \geqslant 0$ is given by

$$
p\left(S_{m, j} \mid S_{\boldsymbol{\theta}, j}, \sigma\right)=\frac{S_{m, j}}{\sigma^{2}} \exp \left(-\frac{S_{m, j}^{2}+S_{\boldsymbol{\theta}, j}^{2}}{2 \sigma^{2}}\right) I_{0}\left(\frac{S_{m, j} S_{\boldsymbol{\theta}, j}}{\sigma^{2}}\right) .
$$

Here $S_{\boldsymbol{\theta}, \boldsymbol{j}}$ denotes the true underlying value given the parameter vector $\boldsymbol{\theta}, \sigma$ the standard deviation of the noise, and $I_{0}$ the zeroth order modified Bessel function of the first kind. Now the $\log$ likelihood function $\ln L\left(\boldsymbol{\theta}, \sigma \mid \mathbf{S}_{\mathbf{m}}\right)$ becomes

$\ln L\left(\boldsymbol{\theta}, \sigma \mid \mathbf{S}_{\mathbf{m}}\right)=-2 \ln (\sigma)+\sum_{j=1}^{N_{g}}\left(\ln \left(S_{m, j}\right)-\frac{S_{m, j}^{2}+S_{\theta, \mathbf{j}}^{2}(\boldsymbol{\theta})}{2 \sigma^{2}}+\ln I_{0}\left(\frac{S_{m, j} S_{\boldsymbol{\theta}, \boldsymbol{j}}(\boldsymbol{\theta})}{\sigma^{2}}\right)\right)$.

where the DWIs are assumed independent, such that the joint probability density function of the signal profile $\mathbf{S}_{\mathbf{m}}$ in a voxel is given by the product of the marginal distributions for the measured signal $S_{m, j}$ in each of the $N_{g}$ diffusion weighted directions $\mathbf{g}_{\mathbf{j}}$. ML estimation of the parameters $\boldsymbol{\theta}$ is obtained by maximizing the $\log$ likelihood function: $\hat{\boldsymbol{\theta}}_{M L}=\arg \left\{\max _{\boldsymbol{\theta}}(\ln L)\right\}$.

We propose to estimate the noise level in a dual tensor model to avoid a bias in the estimated diffusivity of crossing fibers. We assert a model for the diffusion weighted signal $S_{\boldsymbol{\theta}, j}$ consisting of two tensors and an isotropic compartment:

$$
S_{\boldsymbol{\theta}, j}=S_{0}\left\{\sum_{i=1,2} f_{i} \exp \left(-b_{j} \mathbf{g}_{\mathbf{j}}^{\mathbf{T}} \mathbf{D}_{\mathbf{i}} \mathbf{g}_{\mathbf{j}}\right)+f_{\text {iso }} \exp \left(-b_{j} D_{i s o}\right)\right\},
$$

where $S_{0}$ is the signal measured without diffusion weighting and $f_{\text {... }}$ the volume fractions, with $f_{2}=1-f_{1}-f_{\text {iso }}$. We choose $D_{\text {iso }}=3.0 \cdot 10^{-3} \mathrm{~mm}^{2} \mathrm{~s}^{-1}$ as an isotropic diffusion constant (to approximate diffusion values reported in CSF), because $f_{i s o}$ and $D_{i s o}$ are dependent. The signal is measured with a diffusion weighting $b_{j}$ in gradient direction $\mathbf{g}_{\mathbf{j}}$. The diffusion tensors are spectrally decomposed, $\mathbf{D}_{\mathbf{i}}=\mathbf{R}_{\mathbf{i}} \mathbf{E}_{\mathbf{i}} \mathbf{R}_{\mathbf{i}}$, with $\mathbf{E}_{\mathbf{i}}=\operatorname{diag}\left(\lambda_{\|} \lambda_{i \perp} \lambda_{i \perp}\right)$ being the eigenvalue matrix with axial and planar diffusion values (assuming axially symmetric tensors). The two rotation matrices $\mathbf{R}_{\mathbf{i}}$ are parametrized using Euler angles.

The estimation is done per voxel in two steps, by optimizing the log-likelihood function (Eq. 3). First, $\sigma$ is estimated in which we constrain the $\mathbf{E}_{\mathbf{i}}$ to be equal while pertaining constant $f$... and $D_{i s o}$, to reduce the number of parameters and hence the variance in the estimated $\sigma$. Second, all parameters are estimated, with the estimated $\sigma$ substituted in Eq. 3 . 


\subsection{Adaptive Filtering}

Instead of a uniform filter we propose a filter $d_{p}$ that adapts its shape automatically to the local structure ( $p$ indexes the filter components). The filter coefficients are the product of an isotropic Gaussian kernel $G_{p \mid k}$ defined at the central pixel $k$ having a width $\sigma_{G}$, and a normalized weighting based on the single tensor similarity at position $p\left(D_{s, p}\right)$ compared to the tensor in the central pixel $k\left(D_{s, k}\right)$. We represent the six unique single tensor components by a vector $D_{s}$. The filter $d_{p}$ yields

$$
d_{p}=\frac{D_{s, p}^{T} D_{s, k}}{\left|D_{s, p}\right| \cdot\left|D_{s, k}\right|} \cdot G_{p \mid k}\left(\sigma_{G}\right)
$$

in which $|\cdot|$ is the vector norm. Note that in a region of crossing fibers, the DWIs are filtered within the plane of the crossing, spanned by an oblate single tensor. We chose a Gaussian kernel width $\sigma_{G}=4 \mathrm{~mm}$, coarsely corresponding to the uniform filtering neighbourhood of size $1 \times 1 \mathrm{~cm}^{2}$ in 2 .

The adaptive filter was implemented in Matlab (The MathWorks, Natick, $\mathrm{MA}$ ) and is illustrated in figure 1(a) in which the filter kernel is overlaid in red over an image containing the corpus callosum (CC). The original approach involved an isotropic kernel, due to which the filtering mixes white matter and cerebrospinal fluid (left image). The adaptation to the local structure (such as in [11]) of the corpus callosum reduces this effect (right). In crossings, the kernel gets an oblate shape (bottom).
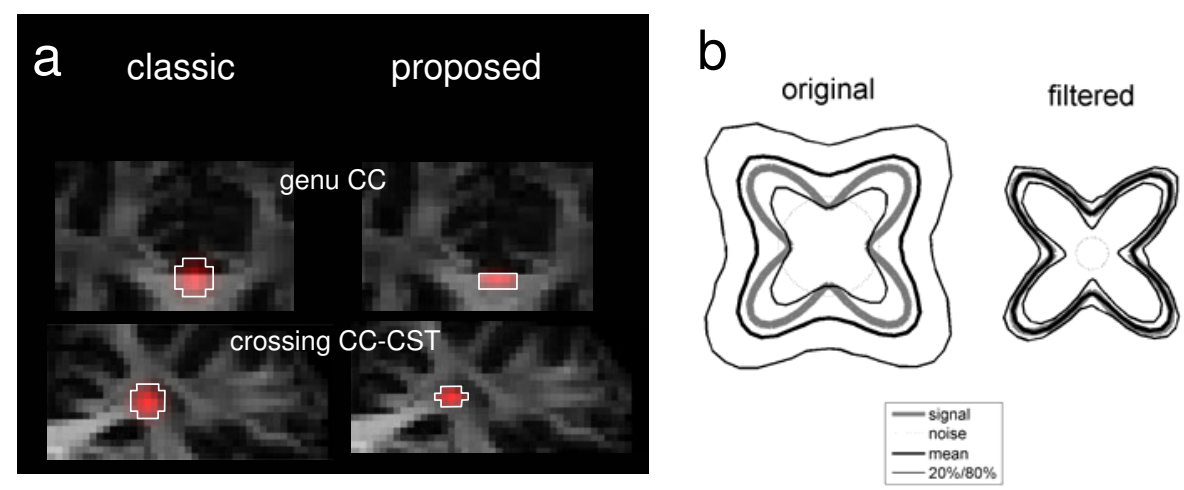

Fig. 1. (a) The original isotropic Aja-Fernandez kernel (left) and the proposed adaptive kernel (right) are shown in red (and manually outlined in white), in the genu of the CC (top) and crossing of CC and CST (bottom). (b) Original and filtered profile of a simulated measurement. 


\section{Experiments}

\subsection{Brain Data Acquisition}

Diffusion Weighted Image acquisition of 20 axial slices (mid-brain) of a healthy volunteer was performed on a Philips Intera 3.0 Tesla MRI scanner by means of a spin echo EPI-sequence, on an eight-channel head coil with SENSE reconstruction. An imaging matrix of $112 \times 110$ was used, from which $128 \times 128$ sized slices were reconstructed, with a voxel size of $1.7 \times 1.7 \times 2.2 \mathrm{~mm}^{3}$. The diffusion weighting was along 92 three-fold tessellated icosahedric gradient directions, with two $b$-values: $\mathbf{b}=\left[\begin{array}{lll}1.0 & 3.0\end{array}\right] \cdot 10^{3} \mathrm{~mm}^{-2} \mathrm{~s}$. Per $b$-value, one non-diffusion weighted image $S_{0}$ was acquired. 20 axial slices were acquired, resulting in a total scanning time of 30 minutes. The deformations induced by eddy currents were corrected with an affine registration in the phase encoding direction [12. In addition, a rigid registration of the $S_{0}$-images and coregistration of the DWIs corrected for patient head motion (up to 2 voxels).

\subsection{Filtered Data Distribution}

The distribution of the data after filtering must be known to accurately estimate the model parameters. Since it was stated that the distribution of the data after filtering may no longer be Rician distributed [2], we emperically studied two potential effects on the distribution. First, the mixing of Rician distributed data might induce a multivariate Rician distribution. Second, the Rician distribution might converge to a Gaussian due to the lower noise level and as result of averaging noisy realizations, in accordance to the Central Limit Theorem. Clearly, if the post-filtering distribution is multivariate Rician distributed, the ML estimation with the given Rician noise model is no longer valid.

The filter's effect on synthetic Rician distorted data was studied with the following procedure. Different FA-values for both tensors $\mathrm{FA}_{1}=0.66$ and $\mathrm{FA}_{2}=0.75$ were selected by constraining axial diffusivities $\lambda_{1 \|}=\lambda_{2 \|}=1.4 \cdot 10^{-3} \mathrm{~mm}^{2} \mathrm{~s}^{-1}$ and planar diffusivities $\lambda_{1 \perp}=0.4 \cdot 10^{-3} \mathrm{~mm}^{2} \mathrm{~s}^{-1}$ and $\lambda_{1 \perp}=0.3 \cdot 10^{-3} \mathrm{~mm}^{2} \mathrm{~s}^{-1}$. Furthermore, we choose $f_{1}=0.4$ and $f_{\text {iso }}=0.15$ with $D_{\text {iso }}=3.0 \cdot 10^{-3} \mathrm{~mm}^{2} \mathrm{~s}^{-1}$ while the opening angle between the long axis of both tensors was $\frac{2 \pi}{5} \mathrm{rad}=72^{\circ}$. The magnitudes of the DWI simulated with these parameters were replicated on a 2D-grid. The signals on each grid point were independently distorted by Rician distributed noise $\left(\mathrm{SNR}=\frac{S_{0}}{\sigma}=1\right)$, such that a significant bias due to the noise was introduced. The experiment was repeated 100 times. The aggregate signal values before and after filtering using the proposed method are shown in figure1(b). It turns out that the filter removes the bias from the data.

In brain data, we focused on a set of highly attenuated DW-signal values, in which a Rician distribution is expected. We included voxels with an anisotropic diffusion profile, by thresholding on $\mathrm{FA}_{¿} 0.5$ and $\mathbf{M D}>1.0 \cdot 10^{-3} \mathrm{~mm}^{2} \mathrm{~s}^{-1}$. Per voxel the DW-signal value $S_{j}$ was selected from the signal profile $\mathbf{S}_{\mathbf{m}}$ whose corresponding gradient direction $\mathbf{g}_{\mathbf{j}}$ was most closely aligned with the principal diffusion direction of a single tensor fit $\mathbf{v}_{\mathbf{1}}$, i.e. $\left\{S_{j} \in \mathbf{S}_{\mathbf{m}} \mid \underset{j}{\arg \max } \mathbf{g}_{\mathbf{j}} \mathbf{v}_{\mathbf{1}}\right\}$. Histograms 

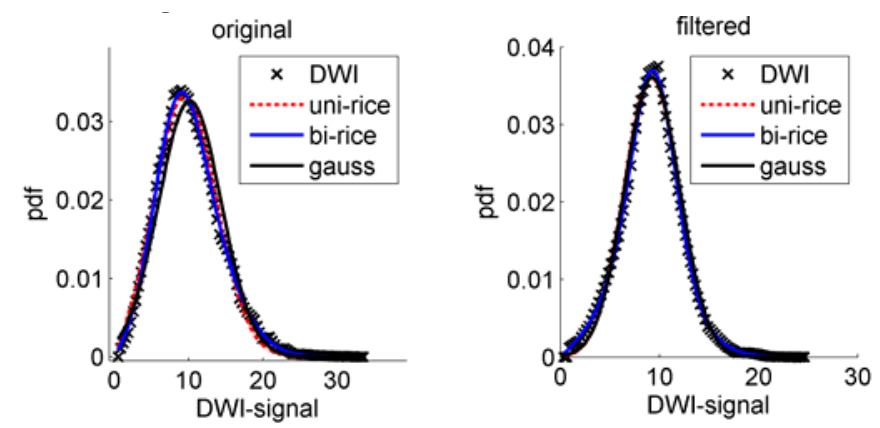

Fig. 2. Histograms of a set of higly attenuated DW-signals values, selected from brain data (see text). A Gaussian (black line), Rician (red line) and bivariate (weighting of two) Rician distributions (blue line) were fit to these points. The left image shows original data, the right image shows filtered data.

before and after applying the proposed noise reduction filter were determined to which uni- and bivariate Rician and Gaussian distributions were fitted: c.f. figure 2. The Kolmogorov-Smirnov test revealed that the Gaussian distribution significantly differed from the measured data, while both Rician distributions comply with the measured data. After filtering, no significant differences were found. This result shows that a univariate Rician is an appropriate noise model prior to filtering. Figure 2 shows that the filtering changes the original noise distribution to a Gaussian. An approximate Gaussian distribution also follows from the Central Limit Theorem. The Rician also fits well due to the higher signal level after filtering: for high SNR the Rician distribution approaches the Gaussian distribution. Practically, a Rician distribution was always fit, since it better generalizes to low signal levels.

\subsection{Accurate and Precise Parameter Estimation}

A Monte Carlo simulation was performed to assess the accuracy of the estimated parameters after noise filtering in the presence of a varying anisotropy in the neighbourhood (see figure 3(a)). A 2D-grid of measurements at crossings was simulated. By adjusting $\lambda_{i \perp}$ the FA-values of both tensors were varied along the horizontal and retained constant along the vertical axis (with a length of 500 voxels). Rician noise was added to all measurements such that the $\mathrm{SNR}=12$. The grid was noise filtered after which we estimated $\mathrm{SNR}=25(\hat{\sigma}=4)$. LevenbergMarquardt optimization of the log likelihood function (Eq. 3) was used in the parameter estimations.

Measurements were performed along the dashed line in figure 3(a). Figure 3(b) shows distributions of the estimated parameters and the mean errors, both before and after noise reduction. The distributions after filtering are narroweras a result of the noise reduction. A significant bias is only observed in $f_{i s o}$, the estimated value is $13 \%$ lower than the modeled value. Simultaneously, the bias in the other 


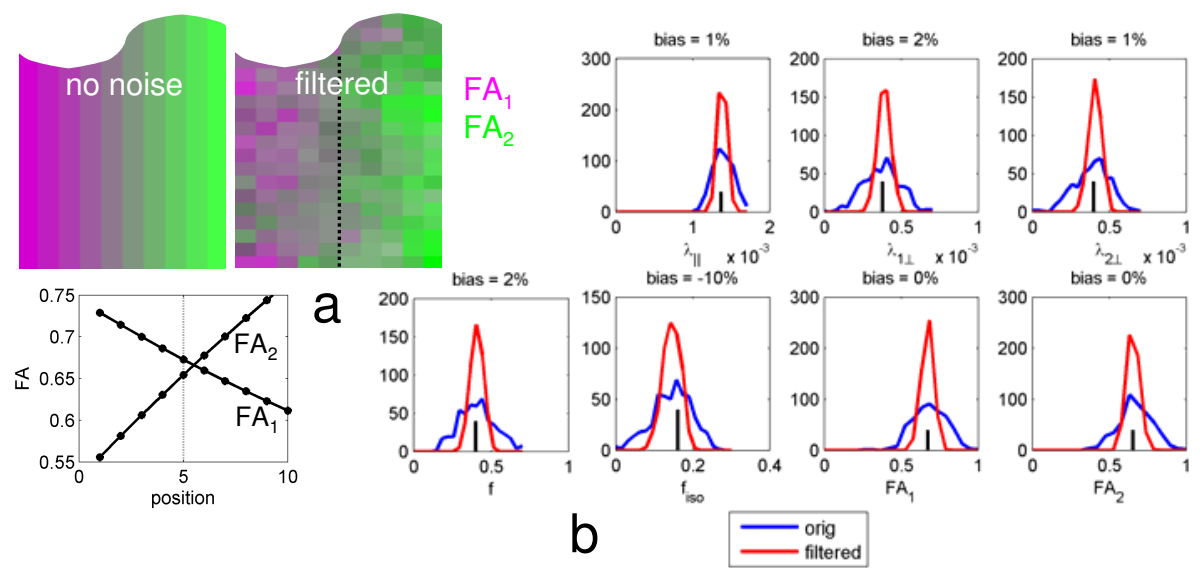

Fig. 3. (a) A 2D-grid of measurements at crossings was simulated. Generated and estimated FA-values are given. Measurements were performed along the dashed line. (b) Distributions of estimated parameters, prior to and after filtering. The true parameter values are indicated by a black bar.

parameters is negligible. We conclude that noise filtering can be safely applied to allow for accurate and precise estimation of diffusivity in crossing fibers.

\subsection{Noise Reduction in Brain Data}

The effect of our noise filtering applied to brain data is illustrated in figure 4 . It demonstrates that the proposed method employing a space-variant adaptive kernel performs better than the original isotropic kernel. This is supported by a shift towards a higher SNR. It should also be noted from the smoothly varying SNR-field that the estimation of $\sigma$ is stable. The average computation time for filtering and tensor estimation per voxel on a notebook computer equals $0.09 \mathrm{~s}$ without filtering, and only $0.17 \mathrm{~s}$ with the proposed method.
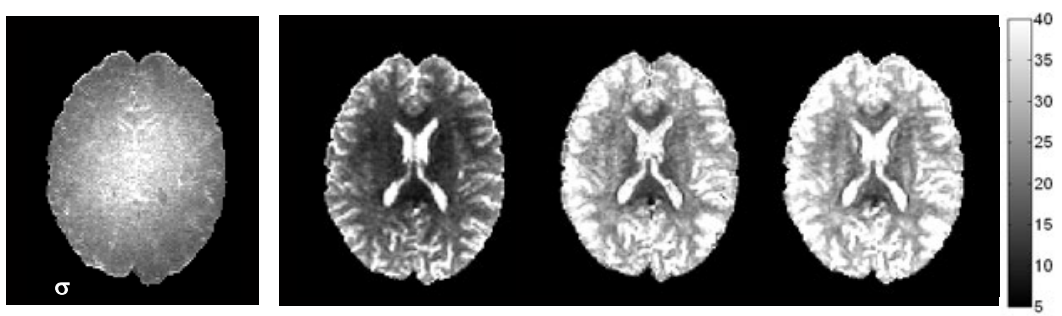

Fig. 4. Estimated SNR $\left(\frac{S_{0}}{\sigma}\right)$ before filtering (left), after filtering with an isotropic kernel (middle), and filtering with the proposed method (right) 


\section{Discussion}

An adaptive filter for suppressing noise in fiber crossings was proposed. The impact on clinical applications of our method is in comparitive studies that aim to assess white matter defects in crossings. Future work will be to estimate a nonstationary noise-covariance for the different DWI directions [13]. The recently proposed Diffusion Type Based similarity measure [14 may even further reduce filtering to homogeneous tissue regions. A crossing fiber phantom whose diffusion properties are accurately known will aid in validating our method.

\section{References}

1. den Dekker, A.J., Sijbers, J.: Advanced Image Processing in Magnetic Resonance Imaging. Signal Processing and Communications, vol. 27, pp. 85-143. CRC Press, Taylor \& Francis Group, LLC, Boca Raton (2005)

2. Aja-Fernandez, S., Niethammer, M., Kubicki, M., Shenton, M., Westin, C.F.: Restoration of DWI data using a rician LMMSE estimator. IEEE Trans. Med. Im. 27, 1389-1403 (2008)

3. Ding, Z., Gore, J., Anderson, A.: Reduction of Noise in Diffusion Tensor Images Using Anisotropic Smoothing. Magn. Reson. Med. 53, 486-490 (2005)

4. Andersson, J.L.: Maximum a posteriori estimation of diffusion tensor parameters using a rician noise model: Why, how and but. Neuroimage 42, 1340-1356 (2008)

5. Landman, B., Bazin, P.L., Prince, J.: Diffusion tensor estimation by maximizing Rician likelihood. In: IEEE 11th ICCV, pp. 2433-2440 (2007)

6. Fillard, P., Pennec, X., Arsigny, V., Ayache, N.: Clinical DT-MRI estimation, smoothing, and fiber tracking with log-euclidean metrics. IEEE Transactions on Medical Imaging 26, 1472-1482 (2007)

7. Basu, S., Fletcher, T., Whitaker, R.: Rician noise removal in diffusion tensor MRI. In: Larsen, R., Nielsen, M., Sporring, J. (eds.) MICCAI 2006. LNCS, vol. 4190, pp. 117-125. Springer, Heidelberg (2006)

8. Martin-Fernandez, M., Munoz-Moreno, E., Cammoun, L., Thiran, J.P., Westin, C.F., Alberola-Lopez, C.: Sequential anisotropic multichannel Wiener filtering with Rician bias correction applied to 3D regularization of DWI data. Med. Im. Anal. 13, 19-35 (2009)

9. Bansal, R., Staib, L., Xu, D., Laine, A., Liu, J., Peterson, B.: Using perturbation theory to reduce noise in diffusion tensor fields. Med. Im. Anal. 13, 580-597 (2009)

10. Caan, M., Khedoe, H., Poot, D., den Dekker, A., Olabarriaga, S., Grimbergen, C., van Vliet, L., Vos, F.: Estimation of diffusion properties in crossing fiber bundles. IEEE Trans. Med. Im. (2010) (in press)

11. van Kempen, G., van den Brink, N., van Vliet, L., van Ginkel, M., Verbeek, P., Blonk, H.: The application of a local dimensionality estimator to the analysis of $3 \mathrm{~d}$ microscopic network structures. In: Proc. 11th Scandinavian Conference on Image Analysis (SCIA), pp. 447-455 (1999)

12. Mangin, J.F., Poupon, C., Clark, C., Bihan, D.L., Bloch, I.: Eddy-current distortion correction and robust tensor estimation for MR diffusion imaging. In: Niessen, W.J., Viergever, M.A. (eds.) MICCAI 2001. LNCS, vol. 2208, pp. 186-193. Springer, Heidelberg (2001)

13. Tristán-Vega, A., Aja-Fernández, S.: DWI filtering using joint information for DTI and HARDI. Medical Image Analysis (2009)

14. Moreno, E.M., Fernández, M.M.: Characterization of the similarity between diffusion tensors for image registration. Computers in Biology and Medicine 39, 251-265 (2009) 